\title{
INFLUENCE DE LA DRÊCHE DE BRASSERIE ET DE Leucaena leucocephala SUR LA CROISSANCE DE THRYONOMYS SWINDERIANUS EN CAPTIVITÉ
}

\author{
A. FANTODJI' ${ }^{1}$, B. TRAORE ${ }^{1}$ ET L.P. KOUAME ${ }^{2}$ \\ ${ }^{1}$ Laboratoire de Biologie et Cytologie Animales, UFR des Sciences de la Nature, \\ Université d'Abobo-Adjamé, 02 BP 801 Abidjan 02, Côte d'Ivoire. \\ E-mail: agatfa@hotmail.com \\ ${ }^{2}$ Laboratoire de Biochimie et Technologie des Aliments, \\ UFR des Sciences et Technologie des Aliments, Université d'Abobo-Adjamé \\ 02 BP 801 Abidjan 02, Côte d'ivoire.
}

\begin{abstract}
RESUME
L'influence de la drêche de brasserie et de Leucaena leucocephala additionnés au complément alimentaire a été évaluée sur la croissance des aulacodes en élevage. 16 aulacodes dont 12 femelles et 4 mâles ont été utilisés pour cette étude. Deux groupes composés de 4 femelles pour 1 mâle ont été soumis au régime alimentaire standard pratiqué à l'aulacoderie expérimentale de l'Université d'Abobo-Adjamé (fourrage : 2 fois par jour, plus une ration de complément alimentaire). Un troisième groupe de 6 animaux a été organisé en 3 lots de 2 animaux chacun. Chaque lot a reçu en plus du fourrage, une ration de complément alimentaire. Aux rations des lots 1 et 3 on a ajouté respectivement de la drêche de brasserie ou de folioles séchées de Leucaena leucocephala. Les animaux du lot 2 servant de témoin, n'ont reçu ni drêche de brasserie ni Leucaena leucocephala. Chaque aulacode a reçu une ration quotidienne faite de $83 \mathrm{~g}$ de complément alimentaire. L'analyse bromatologique des ingrédients alimentaires a révélé une teneur en protéine de $28 \%$ pour la drêche de brasserie contre $24,5 \%$ pour les folioles de Leucaena leucocephala. En ce qui concerne les rations alimentaires, celle du lot 1 contient $13,12 \%$ de PB et 53,90 $\%$ d'extractif non azoté (ENA), celle du lot 2 contient 9,62 \% (P/P) de PB et 59,25\% (P/P) d' ENA. La ration du lot 3 , contient $12,25 \%(P / P)$ de PB et $57,16 \%(P / P)$ de ENA. L'étude de la croissance a révélé un Gain Moyen Quotidien (GMQ) plus élevé pour les aulacodes du lot 1 nourris à la drêche de brasserie que celui des animaux du lot 3 nourris au concentré additionné de Leucaena leucocephala. Les groupes nourris à la ration standard ont présenté en moyenne un GMQ de $9 \mathrm{~g} / \mathrm{J}$. L'analyse statistique de la consommation des différents lots (ANOVA, intervalle de confiance de $95 \%$ ) n'a décelé aucune différence significative. II ressort de cette étude que la ration additionnée de drêche de brasserie a donné les meilleures potentialités nutritionnelles par rapport à Leucaena leucocephala.
\end{abstract}

Mots clés: Elevage, Aulacode, Influence, Drêche de brasserie, Leucaena leucocephala, Côte d'ivoire

\author{
ABSTRACT \\ IMPACT OF BREWERY REFUSE AND LeUcaena leucocephala AS FOOD SUPPLEMENT \\ ON CANE RAT GROWTH UNDER BREEDING CONDITIONS
}

The impact of brewery's on cane-rat growth refuse and Leucena leucocephala added to food supplement was tested on sixteen cane-rat were used for the experiment. Ten animals were submitted to a standard food ration. Six of the animals were organized in three groups of two animals per group. A food ration made of a brewery refuse was used for the first group. The second group received a food ration consisting of leaves of Leucaena leucocephala. The third group received no food. All animals were fed with grass twice daily and each animal received a daily ration of $83 \mathrm{~g}$ of food supplement. Biochimical analysis of ingredients 
and food components showed a high rate of protein in bewery refuse (28\%) and $24 \%$ for Leucaena leucocephala. Protein contents of different lot's ration is respectively 13,12 (lot 1) ; 9,62 (lot 2) ; 12,25 (lot 3) respectively. The day feeding is calculated. The growth study show an higher GMQ for animals of first lot feeded with bewery refuse add ration.

Keywords : cane-rat, breeding, feeding, growth, brewery, Leucaena leucocephala, $\mathrm{Cl}$

\section{INTRODUCTION}

L'autosuffisance alimentaire demeure un problème pour la plupart des pays de l'Afrique au sud du Sahara. Ce problème est aggravé par: I'utilisation par ces pays d'outils de production peu performants, les conflits socio-politiques et les foyers de tension qui surgissent un peu partout et dont les populations sont victimes. Un sévère déficit en protéine d'origine animale touche toutes ces populations. L'intensification de l'élevage classique des espèces animales conventionnelles n'arrive pas non plus à couvrir les besoins, et ne peut se faire indéfiniment.

Des alternatives sont recherchées pour satisfaire les besoins alimentaires des populations tout en maintenant les bases écologiques de l'agriculture.

L'Afrique regorge dans ses écosystèmes, des ressources naturelles non encore exploitées. Parmi celles-ci figurent les espèces animales non conventionnelles dont l'élevage constituerait non seulement une source de protéine alimentaire, mais aussi une source de revenu, de création d'emploi et de protection de la biodiversité (Fantodji et Mensah, 2000).

Les animaux concernés par cet élevage non conventionnel sont les gibiers. L'aulacode (Thryonomys swinderianus) est l'un des gibiers le plus prisé de l'Afrique de l'Ouest et dont la viande ne souffre d'aucun tabou (Mensah, 1999 ; Mensah, 1992). En effet, la valorisation de cet animal est totale. L'arôme, la saveur, la tendreté de sa viande, en font un mets recherché et payé au prix fort dans les grands centres urbains (Mensah, 1992 ; Mensah, 1999). Les poils calcinés servent de cicatrisant dans le traitement de certaines plaies. Les fèces, prélevées dans l'intestin et le coecum, sont utilisées comme assaisonnement dans certains plats cuisinés Africains.

En plus de ces rôles alimentaires et sanitaires de l'aulacode, l'impact socioéconomique de l'aulacodiculture est très grand en ce sens qu'elle contribue à la lutte contre la pauvreté (Fantodji et Mensah, 2000).

Dans le souci de satisfaire aux demandes sans cesse croissantes de la viande de ce gibier, tout en préservant les populations d'aulacodes sauvages, des essais de domestication ont été conduits dans quelques pays africains (Anonyme 1 , 1992). Mais, l'élevage de l'aulacode en captivité étroite ne pourra se développer que grâce à la maîtrise des problèmes relatifs à son alimentation, sa croissance, sa reproduction et l'amélioration génétique des espèces adaptées à la captivité.

Notre étude a pour objet d'apprécier l'influence du Leucaena leucocephala et de drêche de brasserie (sous produits agro-industriels) utilisés comme compléments alimentaires, sur la croissance des aulacodes en élevage.

\section{MATERIELS ET METHODES}

\section{MATERIEL \\ Matériel animal}

Seize aulacodes (Thryonomys swinderianus) dont 12 femelles et 4 mâles, ont été utilisés pour cette étude. Les animaux proviennent des fermes d'élevage de la Région Centre-Nord de la Côte d'Ivoire (tableaux 1 et 2 ). 
Tableau 1 : Les groupes d'aulacodes géniteurs soumis au régime standard.

Cane rats reproducer subject to a standard food ration.

\begin{tabular}{|c|c|c|c|c|c|c|}
\hline \multicolumn{7}{|c|}{ Paramètres des groupes d'aulacodes } \\
\hline & \multicolumn{2}{|c|}{ Sexe } & \multicolumn{2}{|c|}{ Age (mois) } & \multicolumn{2}{|c|}{ Poids moyen (kg) } \\
\hline & Mâle & Femelle & Mâle & Femelle & Mâle & Femelle \\
\hline 1 & 1 & 4 & 9 & 6 & 2 & 1,6 \\
\hline 2 & 1 & 4 & 9 & 9 & 2 & 1,7 \\
\hline
\end{tabular}

Tableau 2 : Répartition des aulacodes soumis au régime alimentaire additionné de Leucaena leucocephala ou de drêche de brasserie.

Distribution of cane rats subject to different food rations supplemented with brewery refuse and plant residus from leucaena leucocephala.

\begin{tabular}{|c|c|c|c|c|c|c|}
\hline \multirow{3}{*}{$\begin{array}{l}\text { Caractéristiques } \\
\mathrm{N}^{\circ} \text { enclos }\end{array}$} & \multicolumn{6}{|c|}{ Repartition des aulacodes } \\
\hline & \multicolumn{2}{|c|}{ LOT 1} & \multicolumn{2}{|c|}{ LOT 2 (témoin) } & \multicolumn{2}{|c|}{ LOT 3} \\
\hline & $\mathrm{IE}_{1}$ & $\mathrm{IE}_{2}$ & $\mathrm{IE}_{3}$ & $\mathrm{IE}_{4}$ & $\mathrm{IE}_{5}$ & $\mathrm{IE}_{3}$ \\
\hline Sexe & 1 mâle & 1 femelle & 1 femelle & 1 femelle & 1 femelle & 1 mâle \\
\hline \multirow[t]{2}{*}{ Poids (g) } & 2045 & 1480 & 1545 & 1595 & 1260 & 1270 \\
\hline & \multicolumn{2}{|c|}{3525} & \multicolumn{2}{|c|}{3140} & \multicolumn{2}{|c|}{2530} \\
\hline Age (mois) & 8 & 6 & 6 & 6 & 8 & 9 \\
\hline
\end{tabular}

\section{Matériel végétal}

La source de protéine végétale utilisée provient des folioles séchées de Leucaena leucocephala, une légumineuse arbustive de la famille des Mimosacées riche en matières azotées. Mais, qui contenant de la mimosine $(0,34 \%$ à $1,2 \%$ $\mathrm{P} / \mathrm{P}$ ) qui limite son utilisation (Van de Welde, 1991 ; Waitkwait, 1986). Une aire fourragère comportant une graminée (Panicum maximum), des plants de manioc, de maïs, de la canne à sucre, des papayers, des bananiers, et une légumineuse (Leucaena leucocephala), alimente l'aulacoderie de l'Université d'Abobo-Adjamé en divers fourrages verts.

\section{Sous-produit agro-industriel}

La drêche de brasserie provenant de l'usine SOLIBRA (Abidjan) est un sousproduit du malt utilisé dans la fabrication de la bière. Cette drêche a été mise à sécher pendant $48 \mathrm{~h}$ avant d'être distribuée aux animaux.

\section{Infrastructuresaulacodicoles}

Les aulacodères utilisés sont des enclos compartimentés de forme parallelopipedique, de $2 \mathrm{~m}$ de longueur, 1 $\mathrm{m}$ de largeur et $0,5 \mathrm{~m}$ de hauteur. Les 2 compartiments communiquent entre eux par une ouverture carrée de $20 \mathrm{~cm}$ de côté. 
Cette ouverture permet le libre passage des aulacodes d'un compartiment à l'autre et assure une bonne conduite de l'élevage (Fantodji, 1999). Dans chaque enclos, une mangeoire et un abreuvoir servent respectivement à la distribution du concentré alimentaire et à l'abreuvement, une pierre à lécher est destinée à l'usure des dents des animaux.

\section{METHODES}

\section{Constitution des groupes d'aulacodes}

Deux groupes géniteurs composés chacun de 4 femelles et un mâle sélectionnés selon les critères d'âge, de poids et de provenance parentale (Mensah, 1999; Soro, 2000) ont été soumis au régime alimentaire standard pratiqué à l'aulacoderie expérimentale de l'Université d'AboboAdjamé celui-ci consiste en : fourrage : 2 fois par jour, plus une ration de complément alimentaire. Un 3è groupe de 6 animaux a été organisé en 3 lots (lots 1, 2 et $3)$ de 2 animaux chacun. Chaque lot a reçu en plus du fourrage, une ration de complément alimentaire spécifique. Aux rations des lots 1 et 3 ont été additionnées respectivement la drêche de brasserie ou des folioles séchées de Leucaena leucocephala. Les animaux du lot 2 servant de témoin, n'ont reçu ni drêche de brasserie ni folioles de Leucaena leucocephala. Chaque aulacode a reçu une ration quotidienne composée de complément alimentaire de $83 \mathrm{~g}$.

Les groupes 1 et 2 d'aulacodes géniteurs ont été installés chacun dans un enclos. Chaque aulacode des lots 1, 2 et 3 a été installé dans un enclos individuel (tableau 2). Avant la constitution des lots, des tests de gestation ont été effectués afin d'écarter d'éventuelles aulacodines gestantes qui pourraient influencer les résultats par le développement de leurs fœtus.

\section{Rationnement et distribution alimentaire}

La ration alimentaire des animaux a été composée de fourrages verts et de concentré d'aliment. Le concentré a été constitué de maïs, de poudre de coquille d'escargot, de sel de cuisine et de folioles séchées de Leucaena leucocephala ou de la drêche de brasserie, (figure 1, tableau $3)$.

La distribution alimentaire a été effectuée à raison de trois services par jour selon le procédé ci-après : 1 ) - pesée et distribution du fourrage le matin et le soir ; 2) - service du concentré à midi (figure 1b).

\section{Collecte des données et détermination de l'ingestion alimentaire}

Après consommation de l'aliment, les refus alimentaires du concentré et du fourrage ont été collectés et pesés le jour suivant dans la matinée. Les quantités de fourrage et de concentré consommées par les aulacodes ont été déterminées.

L'ingestion alimentaire a été calculée à l'aide de la formule :

$$
\begin{aligned}
& \mathrm{I}=\mathrm{K}_{1} \times \mathrm{AD}-\mathrm{K}_{2} \times \mathrm{AR} \\
& \text { où : } \\
& \mathrm{I} \text { : ingestion alimentaire; } \\
& \mathrm{K}_{1} \text { : pourcentage de matière sèche }
\end{aligned}
$$
de l'aliment distribué ;

$A D$ : masse en matière fraîche de l'aliment distribué ;

$\mathrm{K}_{2}$ : pourcentage de matière sèche du refus alimentaire ;

$A R$ : masse en matière fraîche (MF) du refus alimentaire.

$$
\begin{aligned}
& K_{1}=\left[M_{1}-\left(M_{1}-M_{2}\right)\right] / M_{1} \times 100 \\
& \text { et } \\
& K_{2}=\left[M_{3}-\left(M_{3}-M_{4}\right)\right] / M_{3} \times 100 \\
& \text { Où : }
\end{aligned}
$$




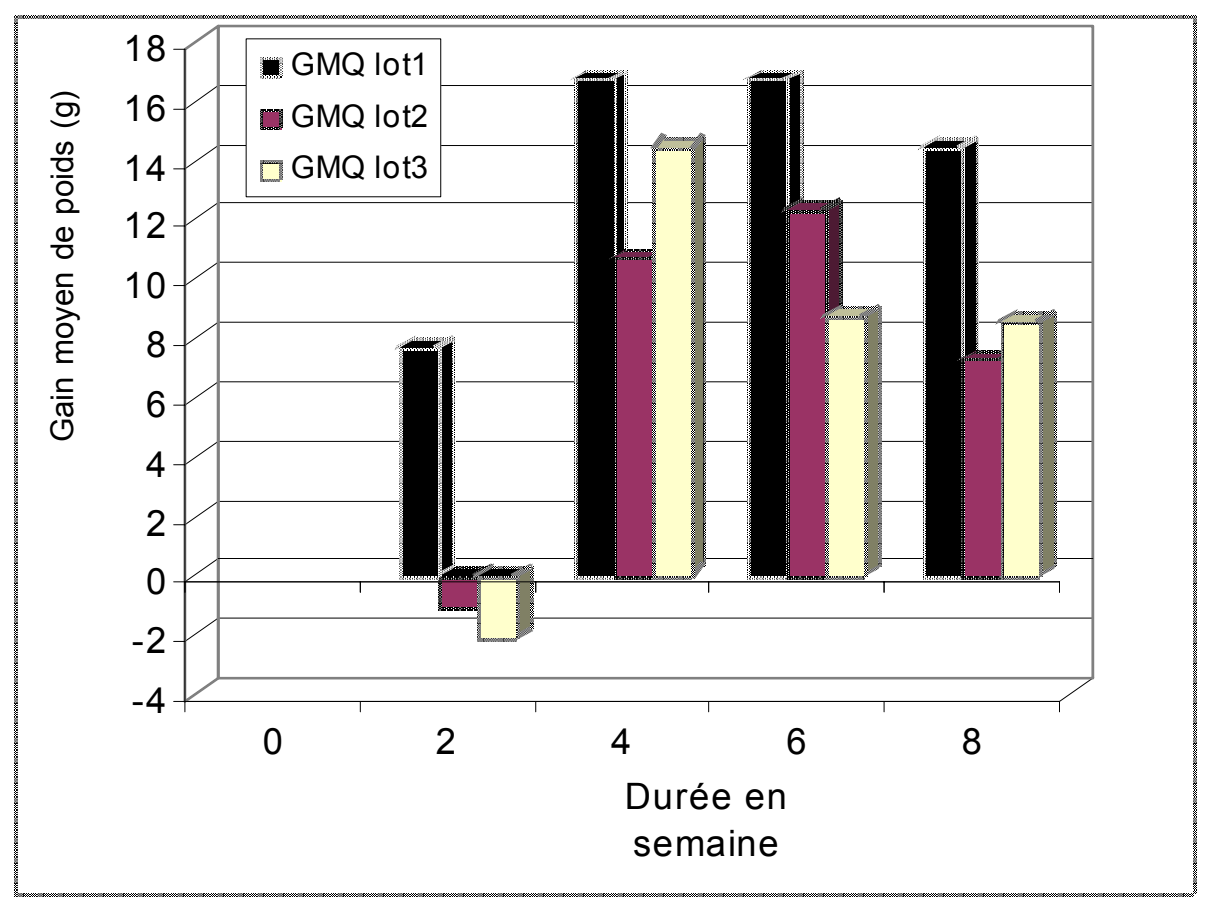

Figure 1 : Evolution des gains moyens quotidiens de poids (GMQ) des aulacodes expérimentaux. Average Daily cane rate weight gains.

Tableau 3 : Composition des rations individuelles des différents lots d'aulacode. Composition of the rations fed individually to cane rats.

\begin{tabular}{lccc}
\hline \multicolumn{4}{c}{ Masse des rations individuelles (g) } \\
\hline Ingrédients & $\begin{array}{c}\text { Lot 1 avec } \\
\text { drêche } \\
\text { sans leucaena }\end{array}$ & $\begin{array}{c}\text { Lot 2 (témoin) } \\
\text { sans leucaena } \\
\text { ni drêche }\end{array}$ & $\begin{array}{c}\text { Lot } 3 \text { avec } \\
\text { leucaena } \\
\text { sans drêche }\end{array}$ \\
\hline Maïs & 70 & 80 & 70 \\
Drêche de brasserie & 10 & 0 & 0 \\
Leucaena leucocephala & 0 & 0 & 10 \\
$\begin{array}{l}\text { Poudre de coquille d'escargot } \\
\text { (Achatina achatina) } \\
\text { Sel de cuisine }\end{array}$ & 2,5 & 2,5 & 2,5 \\
Total & 0,5 & 0,5 & 0,5 \\
\hline
\end{tabular}


$M_{1}$ et $M_{3}$ sont respectivement les masses fraîches de l'aliment distribué et du refus alimentaire.

$M_{2}$ et $M_{4}$ respectivement les masses de matières sèches des aliments ingérés. Les valeurs des ingestions ont été soumises au test statistique ANOVA au seuil de confiance de $95 \%$.

Les aulacodes ont été pesés toutes les quinzaines afin de déterminer l'influence des aliments sur la croissance. Le Gain Moyen Quotidien (GMQ) a été calculé pour chaque aulacode. L'indice de consommation a été calculé selon la formule suivante :

$$
I C=\text { quantité ingérée/ } \mathrm{Pf}-\mathrm{Pi}
$$
vif initial

Avec $\mathrm{Pf}=$ Poids vif final et $\mathrm{Pi}=$ Poids

\section{Analyse bromatologique}

Les compositions chimiques des ingrédients et des rations ont été déterminées selon les procédés préconisés par le ministère de la coopération et du développement (Anonyme 2, 1976). La matière sèche est déterminée après séchage à l'étuve à $130^{\circ} \mathrm{C}$. La matière grasse a été déterminée (Anonyme 2, 1976). La détermination de la teneur en matière azotée a été faite par la méthode préconisée par le Bureau Interprofessionnel d'études analytiques, (Anonyme 3, 1972).

\section{RESULTATS}

\section{CONSOMMATION ALIMENTAIRE}

Les drêches de brasserie et les folioles séchées de Leucaena leucocephala se sont révélées plus riches en protéines que les rations alimentaires (tableau 4).

La consommation alimentaire (ou ingestion), exprimée par rapport à la ma- tière sèche est consignée dans le tableau 5. Les animaux IE1 et IE3 ont présenté les masses moyennes journalières de concentré alimentaire ingéré les plus élevées (tableau 5).

\section{EVOLUTION DU POIDS DES AULACODES}

Au niveau du lot 1 , la croissance du mâle IE1 a été nettement supérieure à celle de la femelle IE2 au cours des 4 premières semaines. Au delà de la 4è semaine, les gains moyens quotidiens (GMQ) tendent à s'équilibrer pour les deux sexes. Le mâle et la femelle ont atteint leur GMQ optimum à la 4è (18 g) et à la 6è semaine $(15 \mathrm{~g})$ avant de connaître une baisse (figure 2 ).

Au niveau du lot 2, les gains moyens quotidiens de la femelle IE3 $(16,4 \mathrm{~g})$ ont été deux fois supérieurs à ceux de la femelle IE4 $(8,2 \mathrm{~g})$. Au delà de la 6è semaine, une chute importante de ces valeurs ( $9 \mathrm{~g}$ et $5 \mathrm{~g}$, respectivement) (figure 3 ) a été constatée. Des résultats semblables ont été observés au niveau des aulacodes du lot 3 . Mais, à partir de la 6è semaine, les gains moyens quotidiens du mâle ont été supérieurs à ceux de la femelle du lot 3 (figures 4 et 5). L'évolution pondérale des animaux dans chacun des 3 lots a montré une nette dominance du lot 1 sur les 2 autres (DP=400 g) (tableau 5). Les gains moyens quotidiens ont également été plus élevés dans le lot $1(13 \mathrm{~g} / \mathrm{j})$ que dans les 2 autres lots où ils n'ont représenté qu'un peu plus de la moitié $(7 \mathrm{~g} / \mathrm{j})$ du poids du lot 1 (tableau 5) .

L'aulacode IE4 du lot témoin a affiché le plus fort indice de consommation $(0,120 \pm 0.037)$, tandis que les aulacodes IE1 et IE2 du lot 1 ont présenté les valeurs d'indice de consommation les plus faibles. 
Tableau 4 : Composition bromatologique des aliments.

Composition of the different food ration and food supplements.

\begin{tabular}{lcccccc}
\hline & \multicolumn{5}{c}{ Composition des aliments (\%) } \\
\cline { 2 - 7 } Echantillons & Humidité & $\begin{array}{c}\text { Matière } \\
\text { sèche }\end{array}$ & $\begin{array}{c}\text { Protéines } \\
\text { brutes }\end{array}$ & $\begin{array}{c}\text { Matière } \\
\text { grasse } \\
(\%)\end{array}$ & ENA & $\begin{array}{c}\text { Cellulose } \\
\text { brute }\end{array}$ \\
\hline $\begin{array}{l}\text { Drêche de } \\
\text { brasserie }\end{array}$ & 16,6 & 83,3 & 28,0 & 6,2 & 7,2 & 10,6 \\
$\begin{array}{l}\text { Leucaena } \\
\text { leucocephala }\end{array}$ & 16,6 & 83,3 & 24,5 & 4,8 & 6,5 & 11,3 \\
$\begin{array}{l}\text { Ration 1 avec } \\
\text { drêche }\end{array}$ & 11,4 & 88,5 & 13,1 & 5,8 & 53,9 & 4,3 \\
$\begin{array}{l}\text { Ration 2 sans } \\
\text { drêche sans }\end{array}$ & 14,8 & 85,1 & 9,6 & 3,4 & 59,2 & 3,3 \\
$\begin{array}{l}\text { Leucaena } \\
\begin{array}{l}\text { Ration 3 avec } \\
\text { Leucaena }\end{array}\end{array}$ & 14,7 & 85,2 & 12,2 & 3,4 & 57,1 & 4,3 \\
\hline
\end{tabular}

Tableau 5 : Ingestion alimentaire journalière du concentré et évolution pondérale des aulacodes.

Daily food intake and weight gains of the cane rats.

\begin{tabular}{|c|c|c|c|c|c|c|}
\hline \multirow[b]{2}{*}{$\mathrm{N}^{\circ}$ enclos } & \multicolumn{2}{|c|}{ Lot 1} & \multicolumn{2}{|c|}{ Lot 2 (témoin) } & \multicolumn{2}{|c|}{ Lot 3} \\
\hline & $\mathrm{IE}_{1}$ & $\mathrm{IE}_{2}$ & $\mathrm{IE}_{3}$ & $\mathrm{IE}_{4}$ & $\mathrm{IE}_{5}$ & $\mathrm{IIE}_{3}$ \\
\hline $\begin{array}{l}\text { Poids vif (g) } \\
\text { Départ }\end{array}$ & 2045 & 1480 & 1545 & 1595 & 1260 & 1270 \\
\hline $\begin{array}{l}\text { Poids vif (g) Final } \\
\text { (après } 4 \text { mois) }\end{array}$ & 2855 & 2130 & 2130 & 1830 & 1660 & 1700 \\
\hline $\begin{array}{l}\text { Variation de } \\
\text { Poids (g) }\end{array}$ & 810 & 650 & 585 & 235 & 400 & 430 \\
\hline $\begin{array}{l}\text { Gain moyen } \\
\text { quotidien (g) }\end{array}$ & 14,64 & 11,60 & 10,44 & 4,19 & 7,14 & 7,67 \\
\hline $\begin{array}{l}\text { Ingérés } \\
\text { alimentaire (g) }\end{array}$ & $\begin{array}{c}36,95 \pm \\
8,23\end{array}$ & $\begin{array}{c}28,31 \pm \\
5,98\end{array}$ & $\begin{array}{c}36,32 \pm \\
7,19\end{array}$ & $\begin{array}{c}28,41 \pm \\
8,89\end{array}$ & $\begin{array}{c}23,80 \pm \\
9,47\end{array}$ & $\begin{array}{c}25,61 \pm \\
10,23\end{array}$ \\
\hline $\begin{array}{l}\text { Indice de } \\
\text { consommation }\end{array}$ & $\begin{array}{l}0,045 \pm \\
0,010\end{array}$ & $\begin{array}{l}0,043 \pm \\
0,009\end{array}$ & $\begin{array}{l}0,062 \pm \\
0,012\end{array}$ & $\begin{array}{l}0,120 \pm \\
0,037\end{array}$ & $\begin{array}{l}0,059 \pm \\
0,023\end{array}$ & $\begin{array}{l}0,059 \pm \\
0,023\end{array}$ \\
\hline
\end{tabular}




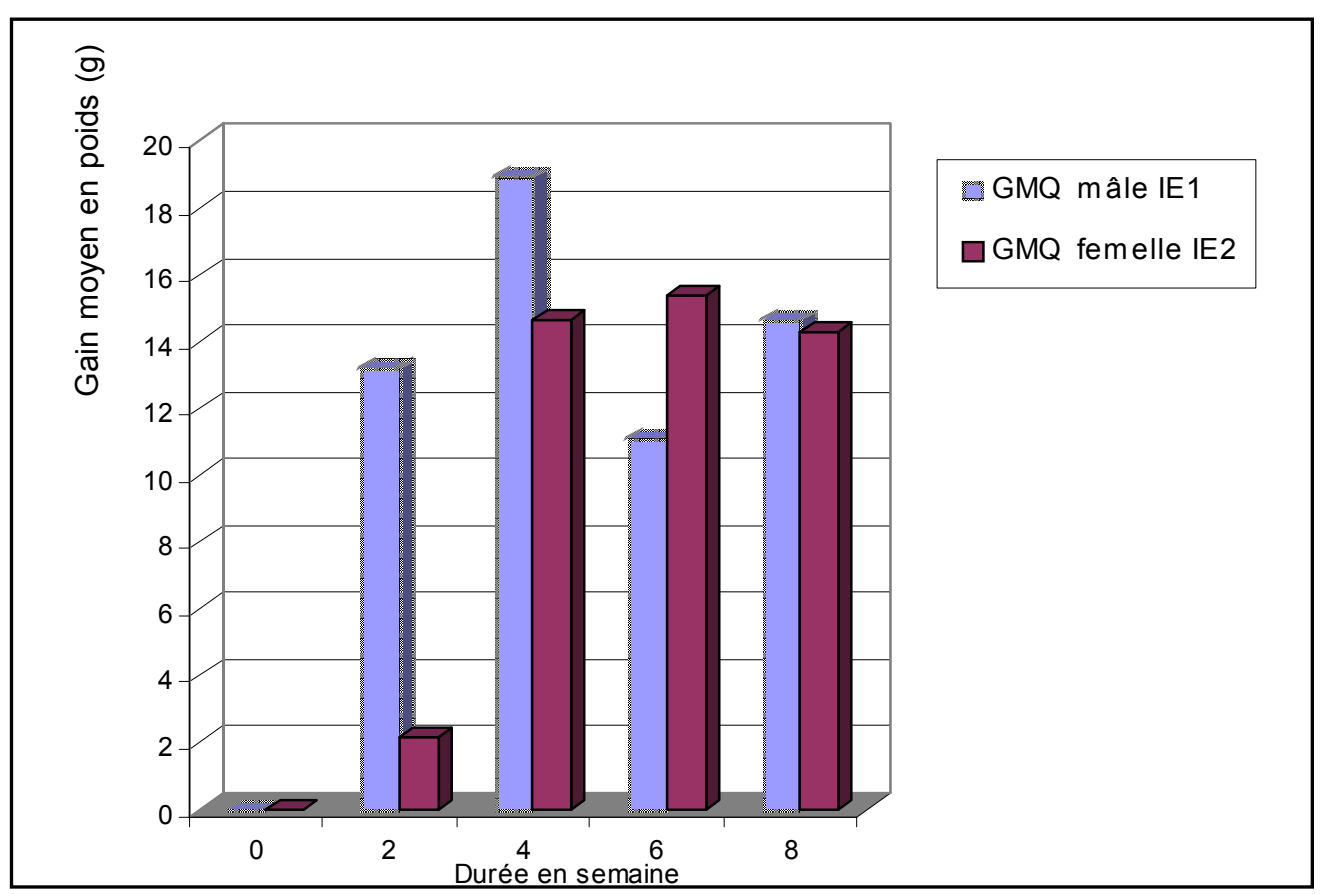

Figure 2 : Evolution des gains moyens quotidiens (GMQ) des aulacodes du lot 1 ayant reçu une ration additionnée de drêche de brasserie.

Average daily weight gains of cane rats from group 1 receiving a ration with breweng refuse added to it.

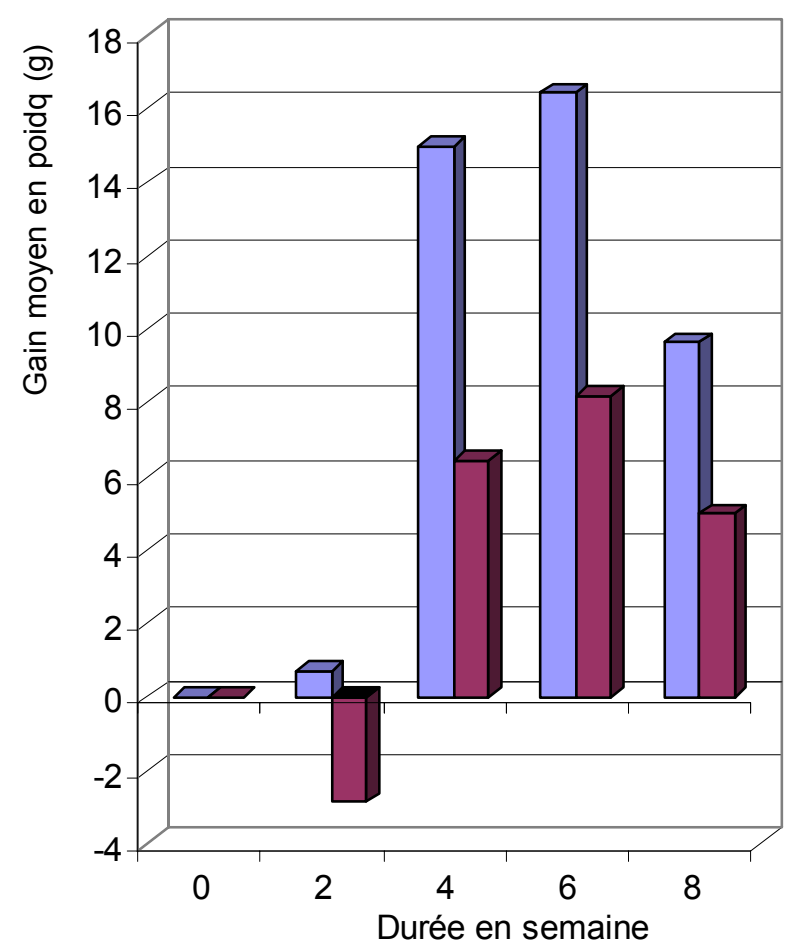

$\square \mathrm{GMQ}$ femelle IE3

$\square \mathrm{GMQ}$ femelle IE4

Figure 3 : Evolution des gains moyens quotidiens (GMQ) des aulacodes du lot 2 (témoin) ayant reçu une ration sans drêche de brasserie ni Leucaena.

Average daily weight gains of cane rats from group 2 (check) receiving a ration with no breweng refuse added to it. 


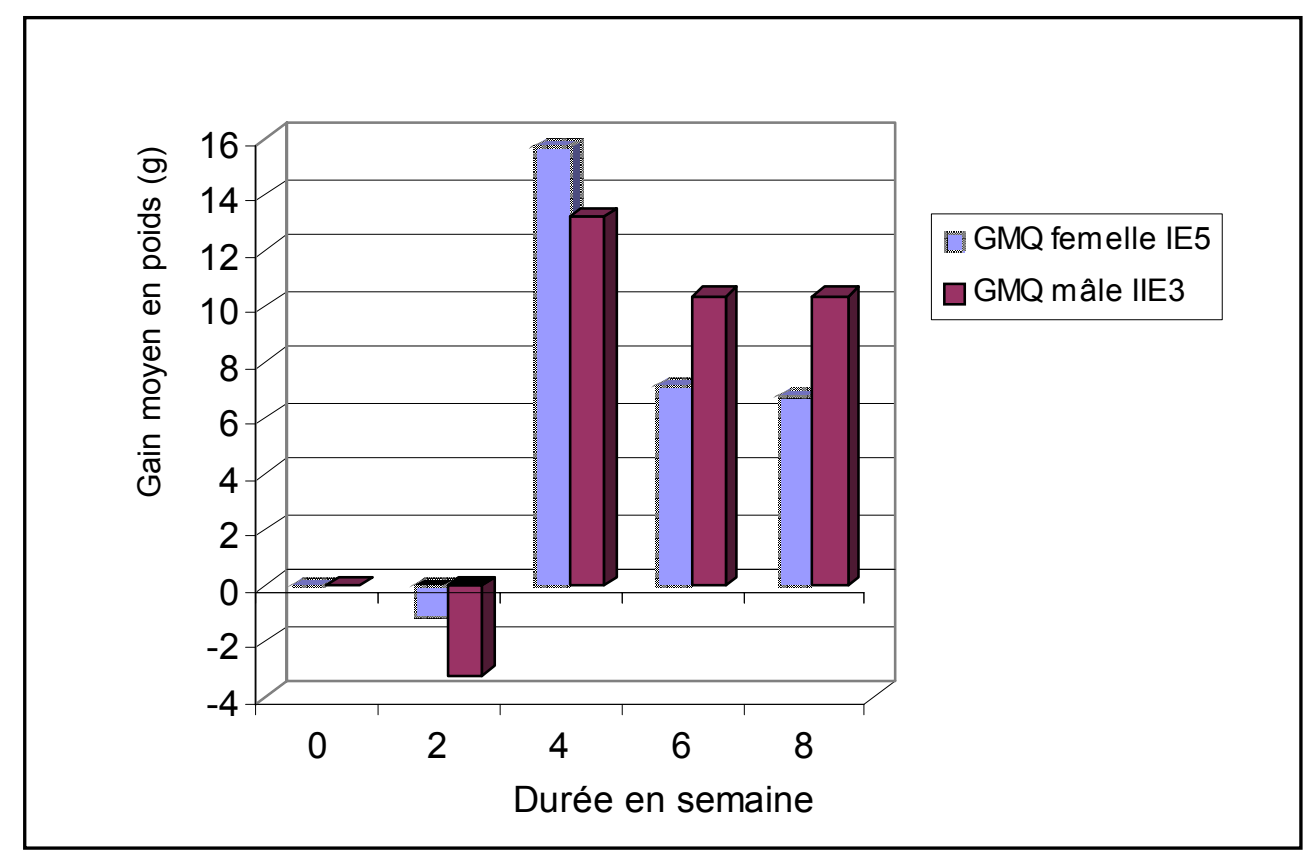

Figure 4 : Evolution des gains moyens quotidiens (GMQ) des aulacodes du lot 3 ayant reçu une ration additionnée de Leucaena.

Average daily weight gains of cane rats from group 3 receivign a ration with leucaena leucocephala added to it.

\section{DISCUSSION}

La composition bromatologique des aliments additionnés de drêche de brasserie et de folioles séchées de Leucaena leucocephala servis aux aulacodes a été semblable à des aliments proposés aux ruminants (Anonyme 6, 1978 et Anonyme $4,1985)$. Une teneur protéique légèrement plus élevée a été néanmoins notée pour la drêche de brasserie, $28 \% \mathrm{P} / \mathrm{P}$, contre $24,5 \%$ P/P pour le Leucaena. L'incorporation de ces ingrédients dans les rations alimentaires a permis de corriger à la hausse les teneurs en protéine des aliments des aulacodes. Ceci a été confirmé par les teneurs en protéine des rations 1 et 3 plus élevées que celles de la ration 2 qui ne contient ni drêche de brasserie, ni folioles séchées de Leucaena leucocephala. Les quantités d'aliment consommés et les valeurs obtenues répondent aux besoins journaliers de l'aulacode en nutriments
(Mensah, 1995). Les valeurs protéiques de ces rations 1 et 3 sont par ailleurs très voisines de celles préconisées par l'Institut de Recherche Agronomique (anonyme $5,1989)$ pour l'alimentation des animaux monogastriques, notamment celle du lapin. Les indices de consommation relativement bas affichés par les aulacodes du lot 1 confirment les GMQ de poids assez élevés présentés par ces animaux par rapport à ceux du lot témoin voie par rapport à ceux du lot 3 .

Dans le lot 2 (témoin) où les aulacodes n'ont reçu ni drêche de brasserie, ni folioles de Leucaena, l'aulacode IE3 a affiché un important gain de poids supérieur à celui de l'aulacode IE4. Ceci pourrait s'expliquer par une consommation journalière par la femelle IE 3 de concentré alimentaire supérieure à celle de la femelle IE4. Les gains moyens quotidiens de poids de ces animaux rapportés à l'ingestion journalière de concentré alimentaire ont 
montré des valeurs semblables pour ces animaux. Ce résultat se traduit par une tendance identique à l'évolution des gains moyens quotidiens de poids de ces aulacodes, avec un optimum commun à la 6è semaine. L'indice de consommation relativement élevé des aulacodes du lot 2 est la preuve de la pauvreté de la ration administrée en éléments nutritionnels.

Au niveau du lot 3, les GMQ des animaux ont été sensiblement égaux. Ces résultats peuvent s'expliquer par le fait que les quantités moyennes journalières de concentré ingéré par les aulacodes de ce lot sont sensiblement égales. Quoique les ingérés alimentaires des aulacodes de ce lot soient inférieurs à ceux du lot témoin (lot 2), leurs indices de consommation ont été plus faibles attestant de l'apport protéique du Leucaena. L'indice de consommation a été encore plus faible avec les animaux du lot 1 soumis à la ration contenant la drêche de brasserie. Ces résultats confirment les valeurs obtenues par les analyses bromatologiques qui ont montré que les rations 1 et 3 , riches en nutriments, ont plus ou moins eu les mêmes composantes biochimiques. Des travaux sur la physiologie digestive (Lawani, 1989) et l'alimentation des aulacodes ont révélé des indices de consommation assez élevés variant entre $1: 6.3( \pm 1.4)$ et $1: 10,5( \pm 1.6)$ (Mensah, 1989); 1:6.7 $( \pm 1,8)$ et 1:10,1 $( \pm 2)$ (Yewadan, 1992). Ces valeurs élevées des indices sont liées au comportement gaspilleur de l'aulacode (Schrage,1988; Mensah, 1989). Mensah (1989; 1992), a signalé que le gaspillage peut être réduit par une granulation des aliments ou par un rationnement.

Dans les conditions naturelles, en plus des apports nutritionnels du complément alimentaire (concentré), l'aulacode pratique la coprophagie, qui est comportement alimentaire permettant à l'aulacode de recycler environ $10 \%$ de ses crottes et de s'approvisionner en vitamines (Holzer et al, 1986). En cas de stress et de perturbations de la digestion (Ewer, 1969), une diminution de la consommation (Anonyme 1,1992 ) et une baisse de la coprophagie sont observées. Cette pratique de coprophagie leur a certainement assuré l'apport en vitamine nécessaire pour une bonne conversion des aliments ingérés, ce qui s'est traduit par les gains en poids observés.

\section{CONCLUSION}

Les folioles séchées de Leucaena leucocephala et la drêche de brasserie ont permis d'améliorer les teneurs en protéine des rations alimentaires 1 et 3 . Ces rations alimentaires ont permis aux aulacodes de connaître de façon significative. La drêche de brasserie et les folioles séchées de Leucaena constituent une source de protéine alimentaire pour les aulacodes.

L'incorporation simultanée de ces deux aliments dans les rations alimentaires peut être d'un apport protéique très appréciable au plan quantitatif et qualitatif.

\section{BIBLIOGRAPHIE}

ANONYME 1, 1992. L'élevage de l'aulacode, Fiches techniques d'élevage tropical, Productions animales, Ministère de la coopération et du développement, Maison Alfort $\mathrm{N}^{\circ} 210 \mathrm{p}$

ANONYME 2, 1976, Recueil des méthodes d'analyse des communautés européennes. Bureau Interprofessionnel d'Etudes Analytiques (BIPEA) $140 \mathrm{p}$

ANONYME 3, 1972, Official Methods of Analysis (13 th edn) Association Officiel Agricultural Chemist (AOAC), Washington D. C.

ANONYME 4, 1985. Table des valeurs des aliments : Composition et valeur alimentaire 
des aliments pour les ruminants, Ministère du Développement Rural et la société allemande de coopération GTZ $20 p$

ANONYME 5, 1991. L'alimentation des animaux monogastriques: porc, lapin, volailles 2è Ed. Institut National de Recherche Agronomique (INRA) Paris Cedex $282 \mathrm{p}$.

ANONYME 6, 1978. Manuel d'alimentation des ruminants domestiques en milieu tropical, IEMVT, Ministère de la coopération Française $525 \mathrm{p}$

EWER (R.F.), 1969. Form and function in the grasscutter thryonomys swinderianus (Rodentia, Thryonomydae) Ghana journal Science vol. 9, pp 131-141

FANTODJI (A.), 1999. Infrastructure Aulacodicole à l'aulacoderie expérimentale de l'Université Abobo-Adjamé (Multigraphié, inédit).

FANTODJI (A.) et (G.A.) MENSAH. 2000. Rôles et impact économique de l'élevage intensif de gibier au Bénin et en Côte d'Ivoire. In Actes séminaire international: I' élevage intensif de gibier à but alimentaire en Afrique. Libreville - Gabon, 23 et 24 mai 2000.p 25-42.

HOLZER, (G.A.) MENSAH, (R.) BAPTIST. 1986. Aspect pratique en élevage d'aulacodes (Thryonomys swinderianus) III- comportement de coprophagie, Rev. Elev. Méd.Vét. Pays tropicaux 39 (2) 247-252.

LAWANI (M.M.). 1989. Physiologie digestive chez l'aulacode (Thryonomis swinderianus): Etude préliminaire. Thèse de Doctorat, E.I.S.M.V. DE L4UNIVERSIT2 CHEIK ANTA DIOP, Dakar (Sénégal), 134 p.

MENSAH (G.A.). 1989. Laufende Untersuchungen am Grasnagerbestad. Rapport d'activité 1988-1989, Université de Hohenheim, $44 \mathrm{p}$.

MENSAH (G.A.). 1991. Manuel d'Aulacodiculture (Elevage d'aulacode) - Edition préliminaire-
Cotonou / Bénin (Multigraphié-Inédit). 50 p.

MENSAH (G.A.). 1991. Elevage des espèces de gibier: cas de l'aulacodiculture (élevage de l'aulacode Thryonomys swinderianus. Actes du dixième Congrès Forestier Mondial, paris- 1991, Revue forestière Française, hors série,vol.5 p. 301-309

MENSAH (G.A.). 1992. Rapport final des travaux de recherche sur l'alimentation chez l'aulacode (Thryonomys swinderianus) en captivité étroite de 1988 à 1992, in Manuel du programme de formation en aulacodiculture (élevage d' aulacode : Thryonomys swinderianus, TEMMINCK 1827), Cotonou $185 \mathrm{p}$.

MENSAH (G.A), 1995. Consommation et digestibilité alimentaire chez aulacode Thryonomys swinderianus, Tropicultura, P 123-124

MENSAH (G.A). 1999. Manuel du programme de formation en aulacodiculture (élevage d'aulacode : Thryonomys swinderianus, TEMMINCK 1827), Programme PEEANC (INRAB Cotonou) 185 p.

SCHRAGE (R.), 1988. Quelques résultats des expériences faites sur les aulacodes au PBAA. Rapport inédit, PBAA/DEP/MDR/Bénin.

SCHRAGE (R) et (T.Y.) YEWADAN. 1995. Abrégé d' aulacodiculture, Schiftenruhe der GTZ $\mathrm{N}^{\circ} 251$, PG 38-50

SORO (D). 2000 Conduite de l'élevage du grand aulacode (Thryonomys swinderianus) en captivité étroite dans l'aulacodérie expérimentale de l' Université d'Abobo - Adjamé : Données sur la croissance et la reproduction. Mémoire de fin de deuxième cycle. $45 p$.

VAN DE WELDE, 1991. Elevage d'aulacode au Zaïre, publication du service agricole $\mathrm{N}^{\circ} 27$ AGCD, 90 p. 
WAITKUWAIT, 1986. Point sur l'élevage de l' aulacode, compte rendu des résultats obtenus après 2 ans de recherche en COTE d' IVOIRE, in Manuel du programme de formation en aulacodiculture (élevage d' aulacode : Thryonomys swinderianus,
TEMMINCK 1827), Cotonou 185 p.

YEWADAN (T. L.). 1992. Alimentation des aulacodes (Thryonomys swinderianus) élevés en captivité étroite. In Actes 1 ère Conférence sur l'aulacodiculture: Acquis et perspectives. Cotonou, Bénin ,pp143-149. 